\title{
Research on Torsional Vibration in Traction Drive System of High-speed Train
}

\author{
Cao Jiang ${ }^{1, \text { a }}$, Xinying Zhao*2, b ${ }^{2}$ Xiangfei $\mathrm{Li}^{1}$, Fei Lin ${ }^{2}$, Zhongping Yang ${ }^{2}$ \\ ${ }^{1}$ CRRC Qingdao Sifang Co., Ltd., Qingdao, 266000, China \\ ${ }^{2}$ School of Electrical Engineering, Beijing Jiaotong University, Beijing, 100044, China \\ aemail: caojiang@cqsf.com, bemail: 14121518@bjtu.edu.cn
}

\begin{abstract}
Keywords: Torsional Vibration; Two-mass System; Resonant Frequency; Stability Analysis
\end{abstract}
\begin{abstract}
In high-speed train, since parts of driving medium are not fully rigid, it is common to have fluctuation of rotating speed in different sizes and phase leading to shaft torsional vibration in driving devices for kinds of reasons. Slight vibration can change the torsional stress on axis, which aggravate axial fatigue damage and decrease the service life, severe vibration may result in shaft damage, which have effect on operation security of the train. This paper firstly established two-mass model of drive system and got the transfer function, introduced the mechanism of torsional vibration through theoretical analysis, which proved that torsional vibration is a system stability problem. Then the author established the Simulink model of traction drive system, which proved unstable factor like speed step and load disturbance can result in shaft torsional vibration phenomenon. Finally analyzes influence parameters of vibration frequency and amplitude by simulation.
\end{abstract}

\section{Introduction}

Safety, comfort and quickness are usually the most concern issues for passenger in high-speed train. Traction motor drives load through driving device like gearbox, coupling and so on, for the rigidity of those devices are limited, elastic deformation will result in torsional vibration along the direction of rotation. When the excitation frequency is given or load disturbance is happened, device will storm energy and cause oppositely phased angular deflection. So the angular deflection between motor and load will be enlarged, which will cause shaft torsional vibration in drive system [1].

Torsional vibration originated from application of rolling mill, low-damping mechanical oscillator consists of the long axis and large inertia load, vibration caused by step-input and load disturbance will menace the integrity of system and lower the quality of each component [2, 3]. Torsional vibration may trigger other form of vibration, which will cover itself. Torsional vibration will be observed only when shaft failure because of fatigue accumulation. This paper mainly analyzes the mechanism and influence factors of torsional vibration of two-mass system through theoretical analysis and simulation.

\section{Mechanism Analysis of Torsional Vibration}

Actual system model can be equivalent to a two-mass system because it is not a rigid connection between traction motor and load, and elastic damping elements can be neglected for their rotational inertia are all small compared with full inertial load [4, 5]. So driving system can be simplified into a two-mass torsional vibration model as Fig. 1 based on following assumptions [6, 7]:

(1) without concern the own vibration state of bearing and motor;

(2) Without regard to efficiency of powerdriven transmission and gap of drive system;

(3) Without regard to the elasticity of train axle, gear, gear shaft and so on;

(4) Drive, driving elements are seen as lumped mass. 


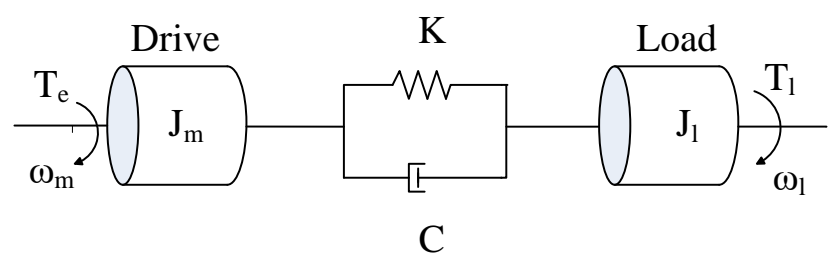

Fig.1. Simplified shaft model of driving system

Mechanic structure is simplified into driving and driven device structure as shown in Fig. 1, motor torque $T_{e}$ is the input stimulation of shaft, differential equations can be established according to Hooke's law and mechanical relation as equation (1).

$$
\left\{\begin{array}{l}
J_{m} \ddot{\theta}_{m}+C\left(\dot{\theta}_{m}-\dot{\theta}_{l}\right)+K\left(\theta_{\mathrm{m}}-\theta_{l}\right)=T_{e} \\
J_{l} \ddot{\theta}_{l}-C\left(\dot{\theta}_{m}-\dot{\theta}_{l}\right)-K\left(\theta_{\mathrm{m}}-\theta_{l}\right)=-T_{l}
\end{array}\right.
$$

In the type: $J_{m}, J_{l}$ are the equivalent rotational inertia of motor-side and load-side, $C$ is the equivalent damping coefficient between driving and driven devices, $K$ is the equivalent torsional rigidity.

Transfer function model of two-mass driving system can be established according to equation (1) as shown in Fig. 2.

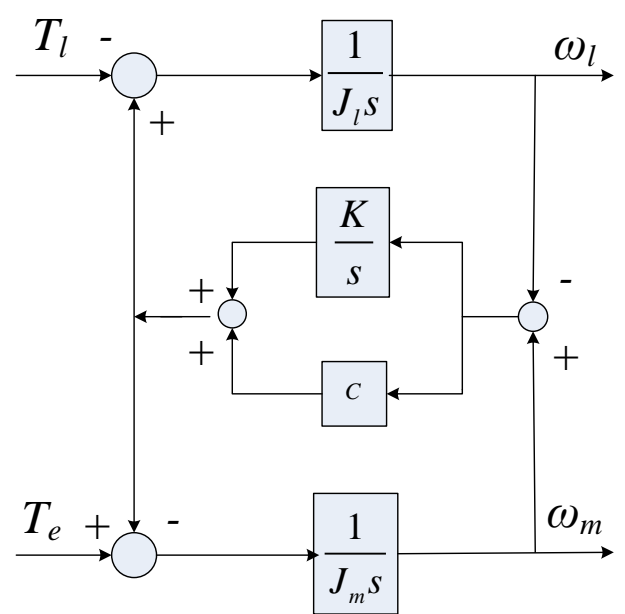

Fig.2. Transfer function model of two-mass driving system

Transfer functions of motor torque to motor speed $\omega_{m}$ and load speed $\omega_{l}$ are:

$$
\begin{aligned}
& G_{\mathrm{m}}(s)=\frac{\omega_{\mathrm{m}}(s)}{T_{\mathrm{m}}(s)}=\frac{1}{\left(\mathrm{~J}_{M}+J_{l}\right) s} \cdot \frac{J_{l} s^{2}+C s+K}{\frac{J_{m} J_{l}}{J_{m}+J_{l}} s^{2}+C s+K} \\
& G_{1}(s)=\frac{\omega_{1}(s)}{T_{m}(s)}=\frac{1}{\left(\mathrm{~J}_{M}+J_{1}\right) s} \cdot \frac{C s+K}{\frac{J_{m} J_{l}}{J_{m}+J_{l}} s^{2}+C s+K}
\end{aligned}
$$

The transfer function $\mathrm{G}_{\mathrm{m}}(\mathrm{s})$ consists of inertial element and second order oscillation element. Second order oscillation element cannot be neglected in medium-high frequency, a dual conjugate complex roots in complex plane is the source of torsional vibration as shown in Fig.3. 


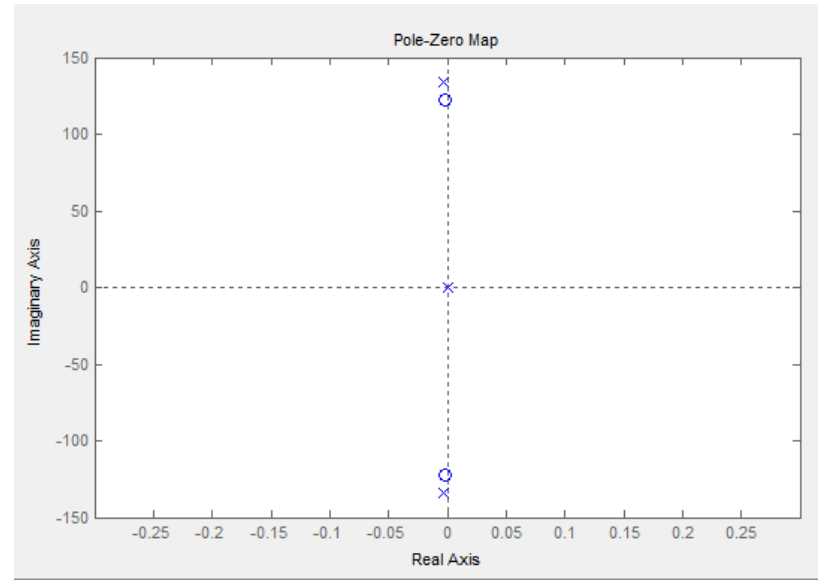

Fig.3. Zero-pole map

Equation (2) can be transformed into equation (4):

$$
\begin{aligned}
& G(S)=\frac{\omega_{m}(S)}{T_{\mathrm{e}}(S)}=\frac{1}{\left(\mathrm{~J}_{M}+J_{l}\right) S} \bullet \frac{1+\frac{2 \xi_{\mathrm{z}}}{\omega_{z}} S+\frac{1}{\omega_{z}^{2}} S^{2}}{1+\frac{2 \xi_{p}}{\omega_{p}} S+\frac{1}{\omega_{p}^{2}} S^{2}} \\
& \omega_{p}=\sqrt{\frac{K\left(J_{m}+J_{l}\right)}{J_{m} J_{l}}}, \quad \omega_{\mathrm{z}}=\sqrt{\frac{K}{J_{l}}} \\
& \xi_{p}=\sqrt{\frac{C^{2}\left(J_{m}+J_{l}\right)}{4 K J_{m} J_{l}}}, \quad \xi_{\mathrm{z}}=\sqrt{\frac{C^{2}}{4 K J_{l}}}
\end{aligned}
$$

In the type: $\omega_{p}, \omega_{z}$ are undamped resonant frequency and antiresonant frequency, $\xi_{p}$, $\xi_{z}$ are resonant and antiresonant damping.

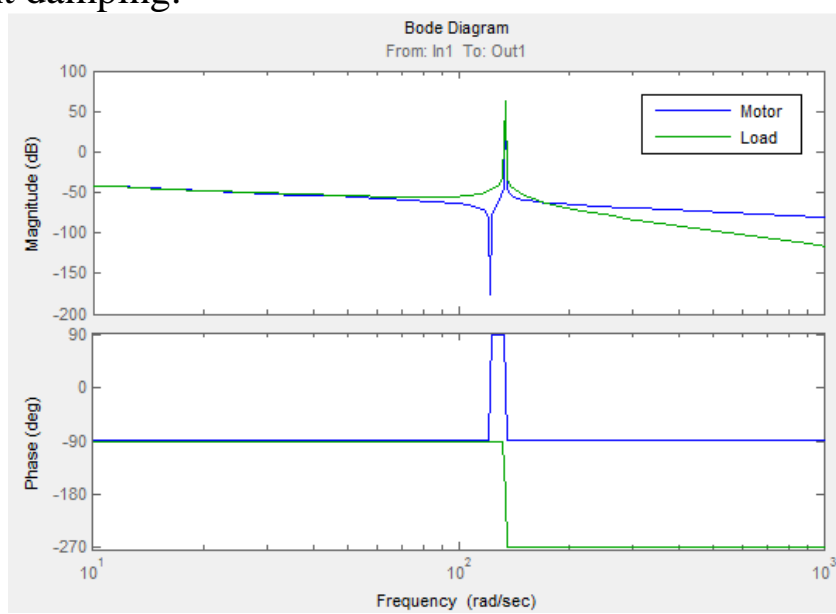

Fig.4. Bode diagram

The two-system model has a resonant frequency and an antiresonant frequency as shown in Fig.4, system gain of motor speed rapid increases to $50 \mathrm{~dB}$ in resonant frequency point $(21.3 \mathrm{~Hz})$, system gain of load speed is much larger compared with motor speed, and the phase position rapid increases in resonant frequency point.

The influence parameters of resonant frequency and amplitude are mainly torsional rigidity $K$ and load rotational inertia $J_{l}$ according to equation (4). 


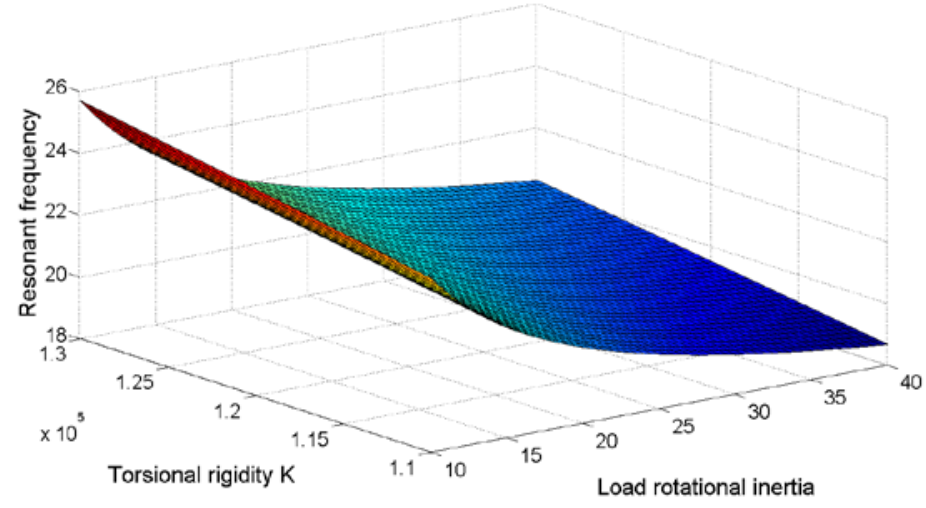

Fig.5. Parameters influence of resonant frequency

\section{Simulation Results of Torsional Vibration Phenomenon}

Traction drive system of high-speed train adopts asynchronous motor vector control, and ensure the stability of speed by using speed close loop, motor control system theory model include mechanical drive as shown in Fig. 6. The simulation is based on the motor control model in CRH2A EMUs, simulate true train operation

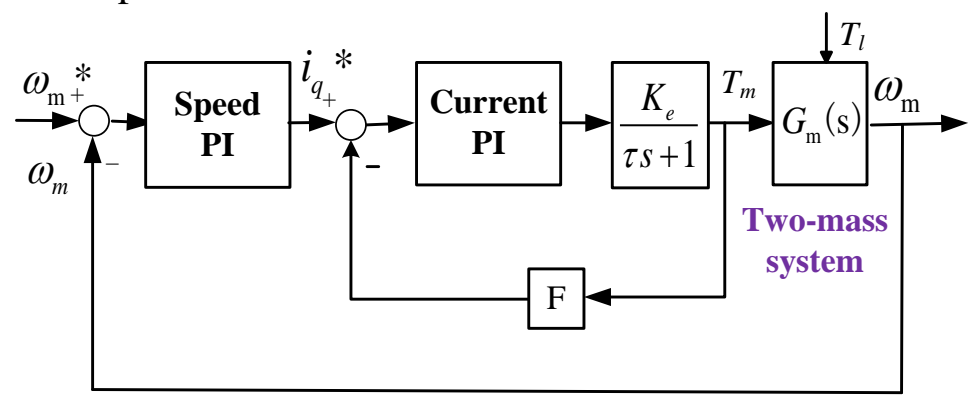

Fig.6. Motor control system theory model include mechanical drive

Bode diagram of load torque to motor speed as shown in Fig.7, it is shown that the resonant frequency is the same with theoretical analysis before, resonant frequency is about $21.3 \mathrm{~Hz}$, system gain still obvious although it decreased from $50 \mathrm{~dB}$ to $14.9 \mathrm{~dB}$ because of speed PI.

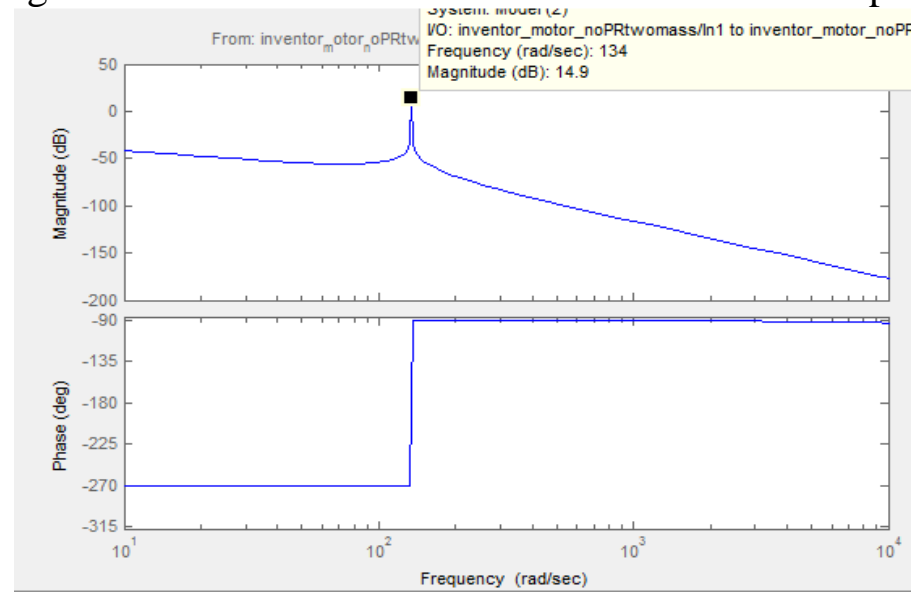

Fig.7. Bode diagram of load torque to motor speed

Setting load torque is $500 \mathrm{~N} \cdot \mathrm{m}$, speed command is $100 \mathrm{~km} / \mathrm{h}, 0-4$ seconds can be seen a speed step in Fig.8. 


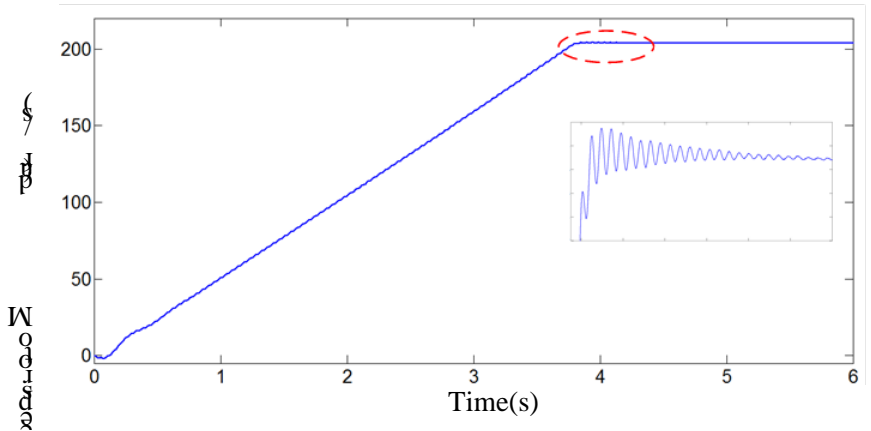

(a) Motor speed

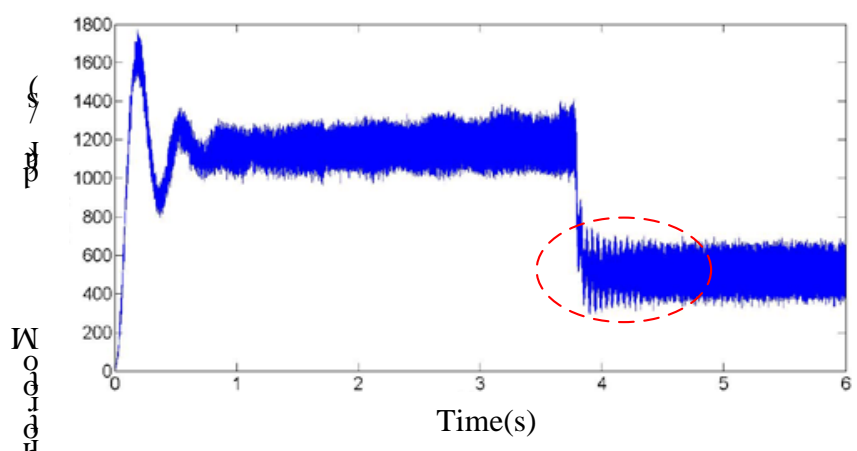

(b) Motor torque

Fig. 8 Motor speed and torque waveforms when load torque is $500 \mathrm{~N} \bullet \mathrm{m}$

It is shown that motor speed and torque can correctly follow speed command in Fig.8, but underdamping resonance phenomenon is obvious, and the resonant frequency is about $21.3 \mathrm{~Hz}$, vibrate lasted about $0.5 \mathrm{~s}$.

When speed goes steady after the 4 second, setting load torque step from $500 \mathrm{~N} \bullet \mathrm{m}$ to $100 \mathrm{~N} \bullet \mathrm{m}$, and observe the torsional vibration as shown below.

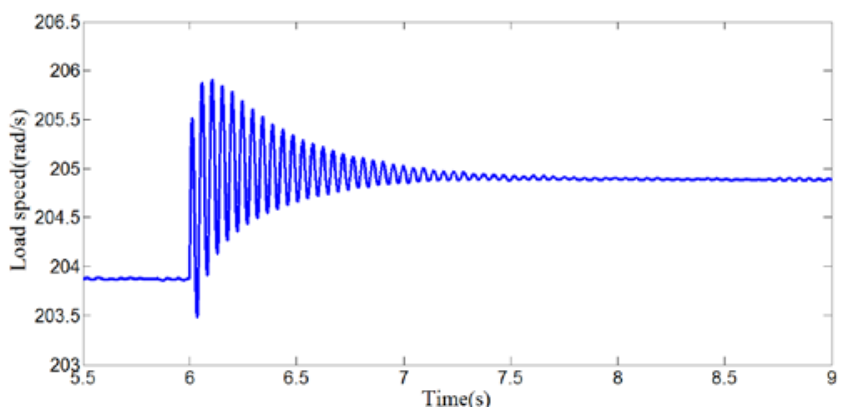

Fig.9 load speed after load torque step

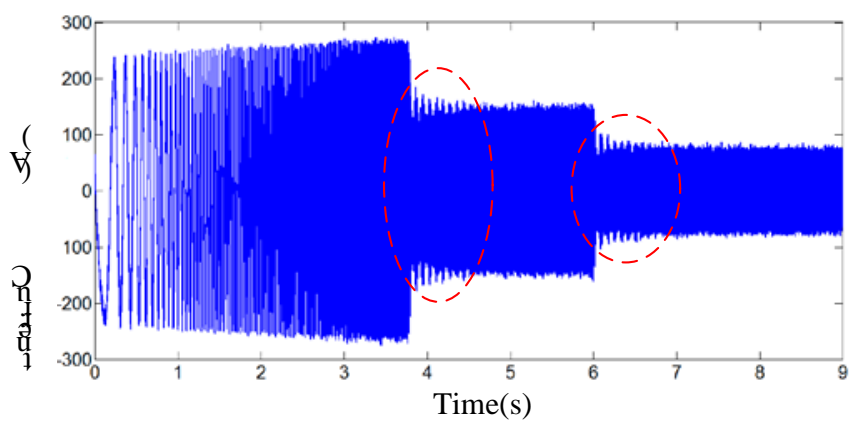

Fig.10 Current after load torque step

It proved that unstable factor like speed step and load disturbance can result in shaft torsional vibration phenomenon, which caused mechanical quantities vibration includes motor-load speed, angle deviations, speed and torque ripple, electrical quantities vibration includes speed PI and current amplitude. 


\section{Parameter influence of torsional vibration}

Resonant frequency is mainly related to load rotational inertia $J_{l}$ and this study is mainly related to performance of speed PI, so this paper researched the parameter influence includes parameter $k_{p}$ of speed PI and load rotational inertia $J_{l}$.

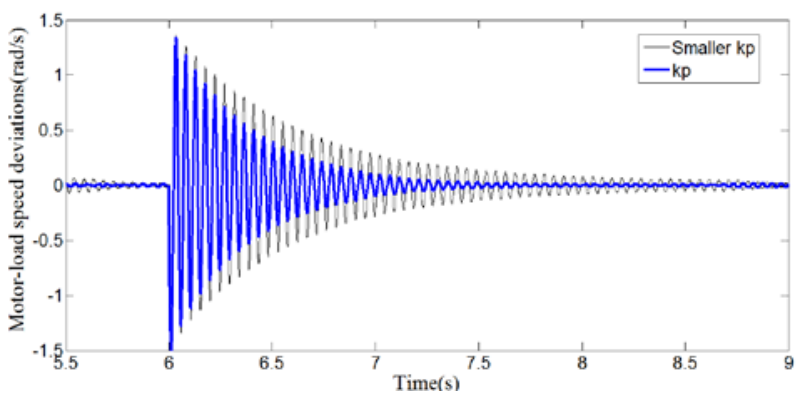

Fig.11. Vibration comparison of different $k_{p}$

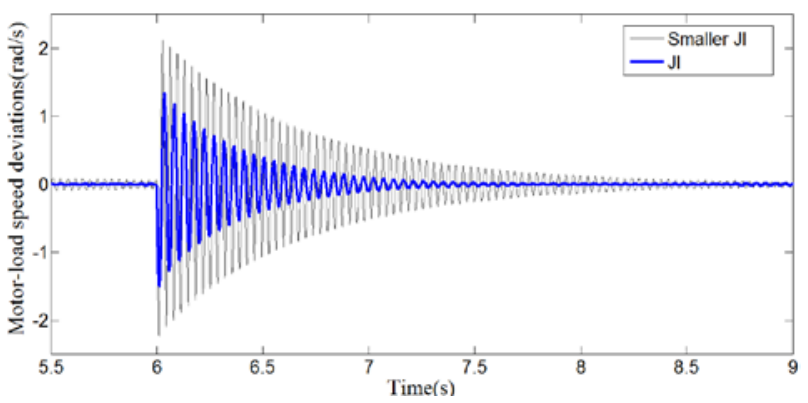

Fig.12. Vibration comparison of different $J_{l}$

Take motor-load speed deviations as an example to analyze torsional vibration, As it is clear from the simulation result in Fig. 11 that vibration duration increased from 1.5 seconds to 3 seconds with $25 \%$ decrement of $k_{p}$. Vibration amplitude of speed deviation increased from 1.2rad/s to $2.1 \mathrm{rad} / \mathrm{s}$ with the $25 \%$ decrement of $J_{l}$ as Fig.13. So $k_{p}$ or $J_{l}$ smaller, vibration more severe and stability rather worse.

\section{Conclusion}

This paper established two-mass model of drive system and introduced the mechanism of torsional vibration through theoretical analysis and simulation, and analyzed influence parameters of vibration frequency and amplitude by simulation. We can get the following conclusions:

(1) Torsional vibration is a system stability problem. Unstable factor like speed step and load disturbance can result in shaft torsional vibration phenomenon.

(2) Torsional vibration is mainly related to load rotational inertia $J_{l}$ and parameter $k_{p}$ of speed PI, $k_{p}$ or $J_{l}$ smaller, vibration more severe and stability rather worse.

\section{References}

[1] Wang yong-qi. Research on Torsional Vibration Suppression of permanent Magnet Synchronous Motor Servo System, Southwest Jiaotong University, 2013.

[2] Gu yi-jiong. Safety Analysis and Application on Torsional Vibration of Turbo-Generator Set.

[M]. Science Press, 2013.

[3] Bao wen, Yang kun. Research on Shaft Torsional Vibration of Turbo-Generator Set. [J]. Turbine Technology, 1998, 40(4): 193-203.

[4] Ma Cheng-bin. Polynomial-Method-Based Design of Low-Order Controllers for Two-Mass Systems. [J]. IEEE Transactions on, 2013, vol.60, no.3.

[5] Krzysztof Szabat. Vibration Suppression in a Two-Mass Drive System Using PI Speed Controller and Additional Feedbacks Comparative Study. [J]. IEEE Transactions on Industrial Electronics, 2007, 54(2):1193-1206.

[6] Yang ming, Hu hao, Xu dian-guo. Cause and suppression of mechanical resonance in PMSM servo system. Electric Machine and Control, 2012, vol.16, no.1: 79-84.

[7] Qiao F, Zhu Q.M, Li S.Y, Winfield A. Torsional Vibration Suppression of a 2-Mass Main Drive System of Rolling Mill with KF Enhanced Pole Placement. [C]. Proceedings of the 4th World Congress on Intelligent Control and Automation, 2002: 206-210. 